\title{
Sensors in Smart Homes for Independent Living of Elderly
}

\author{
Pireh Pirzada \\ University of Southampton \\ Southampton, United Kingdom \\ Email: pireh@idlsys.com
}

\author{
Neil White \\ University of Southampton \\ Southampton, United Kingdom \\ Email: nmw@ecs.soton.ac.uk
}

\author{
Adriana Wilde \\ University of St Andrews \\ St Andrews, United Kingdom \\ Email:agw5@st-andrews.ac.uk
}

\begin{abstract}
-a rapidly ageing population requires support systems which would enable them to preserve dwellers' independence without compromising on their safety or their quality of life. Smart homes for the elderly have the potential to offer unobtrusive health and wellness monitoring. The aim is to provide a safe, independent living environment which can identify and predict problems by monitoring the activities of daily living (ADLs) of the inhabitants. For this, a system able to handle continuous streams of data is required. Such a system can extract the information by using appropriate classification and learning algorithms and thus allow the remote monitoring of health and wellbeing at a high level. The implementation requires: the use of appropriate sensing technologies, identification of ADLs, data pre-processing techniques and machine learning algorithms. This is challenging due to individual differences: such a system must be able to personalize individual needs. Our contribution was the design and implementation of a platform to smartly monitor health condition of elderly using sensor data from a smart home, through an interactive user interface which is user-friendly and multiplatform. This proof-of-concept used off-line data, with the view to extend to real-time data collection in the future, which could then be used to inform support providers remotely.
\end{abstract}

Keywords - Elderly, Smart Homes, Activity recognition, pattern recognition, Smart Home, Unobtrusive monitoring, Health Care, Machine Learning, Learning Algorithms

\section{INTRODUCTION}

In the UK alone, there are approximately 3.64 million people aged 65 and above who live on their own [1]. This number is expected to rise overtime, creating a concern for mental and physical health issues, which is shared amongst families and healthcare professionals. In 2010, it was estimated that 542 million people are aged 60 plus; this is expected to triple by 2050 [1]. Aging can result in slower performance, mental and physical health issues, therefore, creating a concern for their wellbeing. Challenges faced are dementia, diabetes, heart diseases, Alzheimer's disease and other illnesses where the mobility increases with age. Thus, the need for health care services increases, exerting added pressure on the National Health Services as well as private health care providers; increasing the demand for staff and their services, which translates into rises in expenditure. An estimate of $£ 17.4$ billion (two thirds of the overall cost) is paid by people with dementia and their families and $£ 11.6$ billion is spent on private social care. Therefore, creating a rise of fulltime care employment by three times by 2050 [2]. Also, smart homes often rely on the correct use of wearable devices which are widely understood to be inconvenient for this age group. The elderly often object to adopting wearable devices as they add to their cognitive load, hindering performance and may even have a negative effect in their mood [3]. Alternative approaches to circumvent the disadvantages of wearable technologies, often involve the use of video cameras. However this type of surveillance is too intrusive and gives them a perception of being watched $[4,5]$. Other approaches have involved the use of a voice-controlled hub [6], such as those commercially made available in recent times. This approach however, requires being complemented with sensors for effectiveness in smart homes.

Smart home systems facilitate protection of dignity, safety of the individual and independence in their own home. The idea proposes to implement a system that is unobtrusive incooperating technologies such as sensors/IoT devices with cloud integration to handle continuous streams of data and smartly extract the information by using appropriate classification and learning algorithms to enable continuous remote monitoring of health and wellbeing.

Implementing such a system requires identification of ADL, appropriate sensors \& technologies, pre-processing data techniques and learning algorithms to efficiently and accurately detect anomalies in patterns to avoid mishaps, do profiling, connect and alert concerned people assuring their safety. Since each human is different; hence their records vary and their patterns may be different. Thus, a system that adapts to person's activities is required. Visualization of the processed data in a user friendly and summarized manner is required; giving a clear understanding in form of graphs/charts to represent irregularities, patterns and an overall overview of each calendar day. The application should be interactive with good UX (User Experience) using latest technologies available in various operating systems and devices.

The paper after this section is structured as follows: In Section II, we talk about the related work, activities of daily life and its conceptualization. After that comes the technology overview in terms of smart homes for elderly in Section III. In Section IV we talk about the data pre-processing techniques. In Section V, we talk about the pattern recognition specifically KNN and its evaluation. In Section VI \& VII, we talk about the implementation of the system and its features containing details, results and images of the system. Finally, we talk about conclusion and future work in Section VIII. 


\section{LITERATURE REVIEW}

This section starts with the definition of smart homes and related work. It also talks about the activities of daily living and their conceptualization.

\section{A. Smart Homes}

Smart homes for elderly people is associated with the idea of such a system which monitors and logs activities of daily living, identifies pattern, detects anomalies, understands individual human activity, visualizes data and notifies concerned. The main idea is to make certain that elderly person conducts daily living activity as per their routine and ensure safety in terms of identifying and notifying emergency situations. Helping them take care of their health and making living alone independent and safe [6, 7].

\section{B. Related work}

There are a number of relevant projects on smart homes which we have reviewed. Firstly, in Tiger Place, created by The University of Missouri, observed an elderly person's activity and overall health through the use of smart sensors specifically focusing on movements in bed and around the dwelling [8]. Another smart home project, by University of Toronto, collected data from binary sensors installed on house appliances such as switches, bed and tables for assimilating and processing the data. Summarization algorithms were used to identify the changes in the system based on the data collected from sensors in a room [9].

CASAS (Centre for Advanced Studies in Adaptive Systems) is a project run by the University Of Washington State which collects data of people from sensors placed in an apartment that stores the data in files, uses algorithms and techniques to extract patterns and predictions of trends in a smart home $[10,11]$. This dataset has been widely used in the literature of the human activity recognition field [12].

Alam et al. [13] discuss the past, present and future smart home developments and challenges, covering three major concerns around smart homes: comfort, healthcare and security. They discuss the use of heterogeneous devices using middleware to create a distributed network for secure, safe and comfortable home.

Peetoom et al. [14] present literature review on technologies for monitoring and its outcomes for independently living elderly people. They talk about effects of researches conducted for monitoring ADL along with fall detection using various sensors such as PIR motion sensors, body worn sensors, pressure sensors, video monitoring and sound recognition in detail along with their aims of monitoring.

Seo et al. [15] present methods for mining data for ADL using smart home sensors. They talk about controlling sensors and actuators in data driven smart home system with the use of web technologies using commercial sensors to monitor ADL of elderly people in their homes.

García-Hernando et al. [16] present review about different smart home scenarios catering elderly living alone. Various data capturing techniques from sensors along with processing of data, detecting activities using various algorithms have been discussed in this paper. Emphasis on selection of sensors and data processing techniques have been done in this paper to avoid failure of application of smart home.

Lun et al. [17] have also proposed the tracking the activities of daily living (ADL) to facilitate elderly patients in staying at the comfort of their home (and therefore reducing healthcare costs). Their approach involved the use of a combination of a Microsoft Kinect sensor and smart watches for inertial sensing modality. They discuss about the design and implementation of system that tracks human motion using computer vision and inertial sensing.

Chernbumroong et al. [18] proposed an activity recognition system using multi-sensor for monitoring activities of daily living such as exercising, eating, reading, hygiene, walking and watching etc. Various types of wearable sensors were used.

Lara et al. [19] proposed a mobile application system to monitor walking, running, sitting, walking up and down. The data was collected by using smart phone and one sensor device. The data about human activity was being collected on real time basis. Suryadevara et al. proposed another system [20], which monitors home activity to detect and predict behavioral changes of an individual.

Zhuang et al. [21] talks about a system for fall detection in a smart home. He describes his approach for the detection of fall and how it distinguishes particular sounds from other noises. Microphone is used to identify various sounds of fall and using machine learning techniques, Gaussian Mixture Models (GMM) super vector to model noise of fall by applying Euclidean distance to calculate difference among audio segments. Clustering is also done for the audio segments of various types of noises to detect the fall.

Regarding fault detection in daily activities of life in elderly people who are suffering with dementia, Phua et al. proposed that when an error is identified, a visualization or audio is sent to the elderly person to help them enhance their abilities and replace the faded memory [22].

Ordóñez et al. [23] created a system for elderly people who live independently to capture their behavior and analyze it. The system captures measurements using different sensors to collect data of different activities performed by the user and detect the anomaly in the behavior that reflect change in their health status by learning their normal behavior pattern.

Munguia Tapia et al. [24, 25], demonstrated the ease and low-cost of sensor installation for unobtrusive monitoring of user behavior. Their emphasis was on monitoring changes in ADL to detect the emerging medical conditions, collecting data from 2 subjects who lived alone in their apartments.

Nelson et al. [26] also present a system "ActiveLife" using simple motion sensors to track the activities of daily living of mild cognitive patients in their living rooms.

\section{Activities of Daily Living}

In [27] Wilde regards activity recognition mainly from a motion-aware perspective but recognizes that activities of daily living (ADL) include to others not easily detected with motion sensors. ADL is defined by Katz and Lawton which is divided 
into sub-categories: Instrumental, Ambulatory and Transitional. These are helpful in identifying the activity level of a person, and can help detect emergency situations as defined in Table I $[28,29]$.

TABLE I. REGULAR ACTIVITIES

\begin{tabular}{|c|c|c|}
\hline Activity Type & Activities & Sensors \\
\hline Basic & $\begin{array}{l}\text { Bathing, dressing, eating, } \\
\text { drinking, using toilet, taking } \\
\text { medicine and grooming }\end{array}$ & $\begin{array}{l}\text { Environmental, } \\
\text { motion, } \\
\text { Accelerometer and } \\
\text { binary sensors }\end{array}$ \\
\hline $\begin{array}{l}\text { Leisure/ } \\
\text { Instrumental }\end{array}$ & $\begin{array}{l}\text { Using telephone, watching } \\
\text { TV, preparing food, cleaning, } \\
\text { sleeping }\end{array}$ & $\begin{array}{l}\text { Environmental, } \\
\text { motion, } \\
\text { Accelerometer } \\
\text { sensors, heart rate, } \\
\text { presence and door } \\
\text { sensors }\end{array}$ \\
\hline $\begin{array}{l}\text { Ambulatory/ } \\
\text { Other }\end{array}$ & $\begin{array}{l}\text { Lying, sitting, standing, } \\
\text { walking, running, cycling, } \\
\text { walking upstairs, walking } \\
\text { downstairs, lie-to-stand, } \\
\text { stand-to-lie, sit-to-stand, } \\
\text { stand-to sit }\end{array}$ & $\begin{array}{c}\text { Accelerometer } \\
\text { sensor }\end{array}$ \\
\hline
\end{tabular}

\section{Activity Conceptualization}

In [30] Meditskos et al. describes activities can have different level of complexities so they can be broken down in simple steps. One activity can be broken down into multiple activities, for example, going to the toilet. Open door $\rightarrow$ Turn on light $\rightarrow$ use toilet $\rightarrow$ flush toilet.

Sequential activities are defined as activities which are in form of a sequence with time intervals having before and after dependency relations i.e. performed in a sequence. Whereas Concurrent activities are those types of activities which occur in same time span and is possible that activities sharing the same time span may overlap. Interleaved activities mean activities that occur by pre-empting each other. This can be a long and complex form of activity occurring at intervals where one can occur for short amount of time while other can be for longer duration [31].

\section{TECHNOLOGY OVERVIEW}

This section details about the overview of application in terms of sensors/IoT devices and stages of a smart home structure.

Various types of devices, sensors and actuators are available such as Video Cameras, Microphones, RFID, Environmental, Wearable and Simple binary sensors to collect data. These can be connected through Personal Area Network (PAN) or Wireless Sensor Network (WSM). Each type of sensor has its own advantages and disadvantages; hence choosing an appropriate sensor for a specific ADL is important as described in section II.C. Not all sensors are compatible with the same hub or Wireless sensor network, therefore need a middleware connecting various devices leading to a hybrid system $[32,33]$.

\section{A. Application Overview}

The structure of the smart home project consists of four stages which includes sensors installed in a home to monitor daily activities. Communication through gateways to send data to the server containing application for data analytics and storage. The data is filtered using data processing techniques, segmented based on activity and sensor events; as illustrated in Fig. 1.

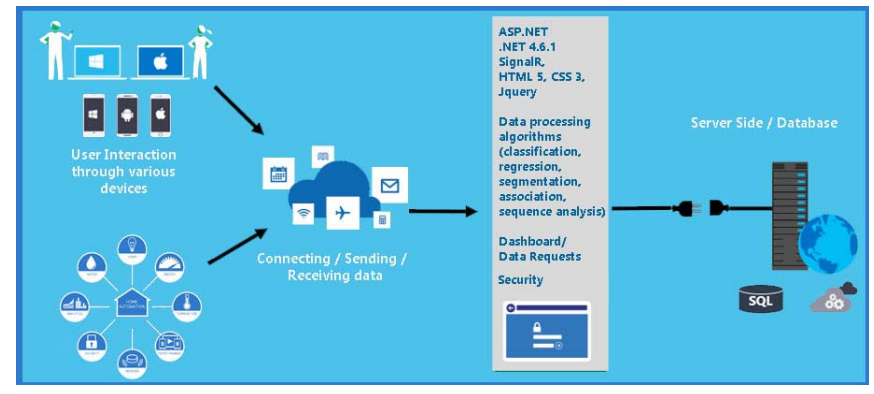

Fig. 1. Architecture of Smart Home Application

\section{B. Smart Home Structure}

Figure 2 illustrates the stages of a smart home structure for implementation of the system.

- Stage 1. This stage covers all the industrial/market sensors/devices available that can be easily installed to collect physiological and clinical data. Hence choosing the appropriate sensors for installation in the house to collect ADL data. $[34,35]$.

- Stage 2. The sensor data is in analogue form which needs to be converted and collected into digital streams for further processing. (DAS) Data acquisition system acts like a middleware between sensor network and the transformed outputs to perform conversion from analogue to digital data, sent through the gateway to send the aggregated digital data to devices/application via wireless (WI-FI) or wired internet (LAN) [36].

- Stage 3. At stage 3, raw data is received by the application server for processing and analysis to convert raw data into information where filtering, clustering and anomaly detection is performed. This stage is crucial and if not managed properly into layers for processing can use up many server resources making application inefficient.

- Stage 4. Once the processing is done in stage 4, next comes the storage of data. This also creates a data warehouse which can be used for predicting patterns from the huge amount of data gathered. ADL can be recorded and behavior patterns can be understood for each individual. The data stored in database can be further processed for depth processing and feedback. Database techniques such as indexing can be used to speed up the database search when needed. The data can be stored on cloud for big data handling as of its rapidly increasing data capabilities. 


\section{PATtern ReCognition}

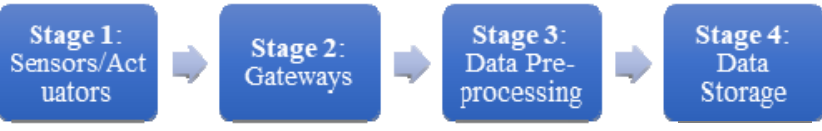

Fig. 2. Stages of smart home structure

\section{DATA PRE-PROCESSING}

Tahani and Sreela [37] argue that data processing is the most time-consuming part of a project, which can take up to 80 percent of its lifetime. Further, real data gathered in the wild using sensors, instruments or processes can present anomalies, errors and sensing failures which means that the data needs to be pre-processed to improve performance.

- Data cleansing techniques are used to find out the required and relevant data. It uses techniques like filling in missing values, remove or level the noise in data and resolving inconsistencies in data.

- Missing values can be handled by using either median or mean, applying data mining techniques to predict the values, using global values or ignoring such fields if possible.

- Data transformation is the process of getting appropriate data in a meaningful form by discarding or clustering methods to remove noisy data, defining new attributes of data, applying aggregating operations on the data, normalizing and generalization of data.

- Data Integration is the process of aggregating different data together by removing conflicts with the existing data. The process of combing data from various sources also known as schema integration.

- Data Reduction is the process of reducing data from large amount to more comprehensive version and representing it. Data Discretization is the reduction of number of values that are of continuous characteristic by dividing the range of attribute intervals.

- Segmenting data is based on activities means identifying the beginning and end-point of an activity. Yoshizawa et al. [38] anticipated method for distinguishing static activities from dynamic ones as activity recognition.

- Feature extracting helps represent the main features from the raw data, improving the efficiency and accuracy of processing algorithms. Once the feature extraction is done, the data is transformed into feature vectors which contain information to be input.

- Feature selection allows finding a relevant data subset from the features extracted vector to reduce noise and increase accuracy of recognition algorithms.
A detailed discussion on different machine learning and data mining techniques used in smart home application were presented in [39, 40, 41]. Artificial intelligence and machine learning techniques can be used to make predictions and learning algorithms e.g. Artificial neural network (ANN), support vector machine (SVM), and K-Nearest Neighbors (KNN) can be used to learn and develop models for the home environment as well as for the behavioral and physiological patterns of the occupants [42].

\section{A. K-Nearest Neighbors (KNN)}

The k-nearest neighbor algorithm $(\mathrm{KNN})$ is a pattern recognition algorithm which is used for classification and regression as well. KNN is instance based learning also known as memory based learning which is learning algorithm that compares input instances with instances in training in machine learning. It simply means that the instances in training set act as knowledge that is already stored in memory and when new instances are provided, it is searched for the most related instance to the new one due to which it is known as lazy learning [41]. The most commonly used distance formula is Euclidean distance (1), (2) though others can be used such as Manhattan distance, Minkowski or hamming distance.

$$
\begin{gathered}
\sqrt{\left(q_{1}-p_{1}\right)^{2}+\left(q_{2}-p_{2}\right)^{2}+\cdots+\left(q_{n}-p_{n}\right)^{2}} \\
\sqrt{\sum_{i=1}^{n}\left(q_{n}-p_{n}\right)^{2}}
\end{gathered}
$$

\section{B. $10 \mathrm{~K}$ fold validation}

Cross validation is also known as rotation estimation which is a validation method for evaluating a predictive model to estimate the accuracy of its performance. The main purpose of cross validation is to define a test dataset to test the model when it is in training stage so, over fitting can be limited. The whole data is known as the full dataset which is divided into training dataset and test dataset [41].

\section{IMPLEMENTATION}

This section talks about the implementation of the system, defining its features with images taken from the system to illustrate visualizations and data.

\section{A. Dataset}

The project uses the MIT's dataset collected by Tapia [24, 25]. The dataset contains data collected from 80 to 100 reed switch sensors installed in two single person apartments for the time period of two weeks. The sensors were installed on daily activity items such as drawers, lights, fridge etc. Table (II) represents each subject's activities that were collected. The dataset for subject 1 was chosen to experiment with for this project as its activities were related to the scope of this project. 
TABLE II. ACtivity Data COLLECTED From SubJects

\begin{tabular}{|l|c|c|}
\hline \multicolumn{1}{|c|}{ Activity } & Subject 1 & Subject 2 \\
\hline Preparing dinner & Yes & Yes \\
\hline Preparing lunch & Yes & Yes \\
\hline Listening to music & No & Yes \\
\hline Taking medication & No & Yes \\
\hline Toileting & Yes & Yes \\
\hline Preparing breakfast & Yes & Yes \\
\hline Washing dishes & Yes & Yes \\
\hline Preparing a snack & Yes & Yes \\
\hline Watching TV & No & Yes \\
\hline Bathing & Yes & No \\
\hline Going out to work & Yes & No \\
\hline Dressing & Yes & No \\
\hline Grooming & Yes & No \\
\hline Preparing a beverage & Yes & No \\
\hline Doing laundry & No \\
\hline Cleaning & Yes & \\
\hline
\end{tabular}

\section{B. Data Pre-processing}

The data provided was filtered for missing values and data redundancy was removed. Data was suitably preprocessed, e.g. the date format was converted from " $m \boldsymbol{m} / \mathbf{d d} / \boldsymbol{y y y y}$ hh:mm:ss" to "yyyy/mm/dd hh:mm:ss" for MS SQL default date format and displayed in the application as " $d \boldsymbol{d} / \mathbf{m} \mathbf{m} / \mathbf{y y y y}$ $\boldsymbol{h h}: \boldsymbol{m m}: \boldsymbol{s s}$ ". The data was categorized by ADL and sensor location of the house. Table III shows Pseudo code implemented in the system for pre-processing data.

TABLE III. PSEUDO CODE FOR PROCESSING DATA

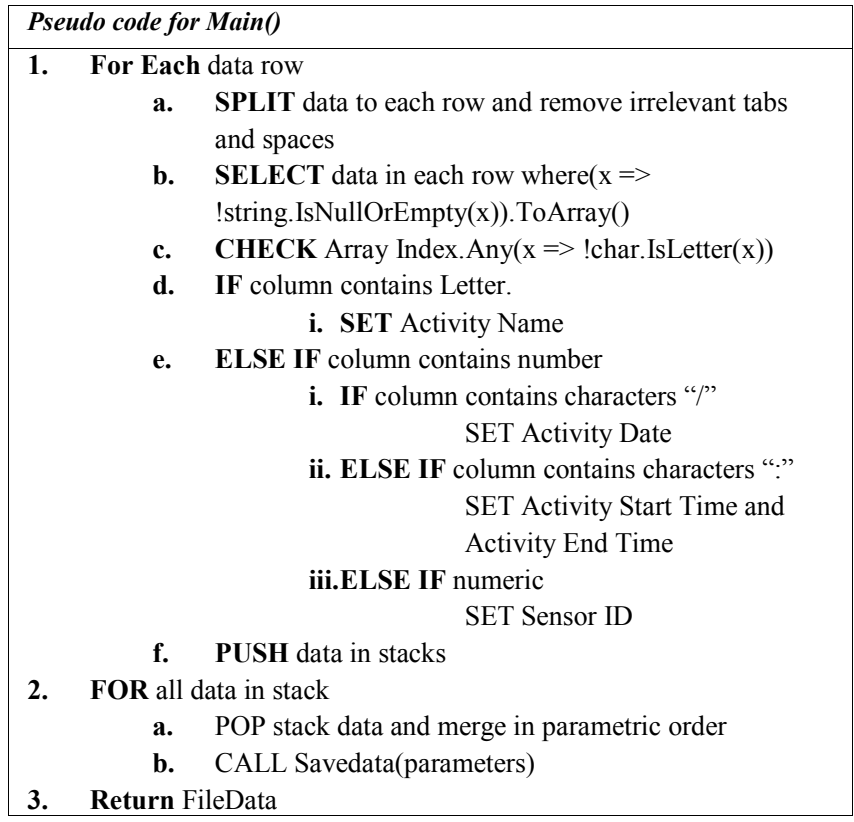

\section{Actor Use Case}

Fig. 3. illustrates various use cases initiated or participated by actors of the system e.g. Dashboard \& Visualizations are accessed by the family member/caretaker who can monitor the ADL of the elderly person. Whereas various sensors gather information and communicate via central Hub to store in database after processing.

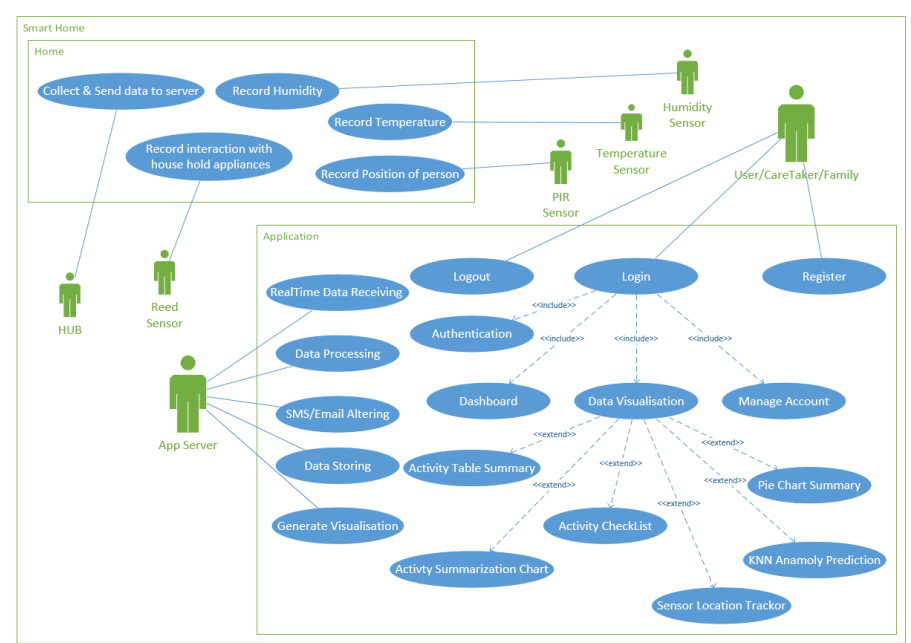

Fig. 3. Use Case Diagram of the system

\section{Anomaly Detection}

The activity diagram (Fig. 4.) illustrates data gathered from sensors in its raw form is pre-processed and analyzed. System generates alerts if any irregular activity occurs and sends a message or a call to the family member/caretaker, along with the location of the elderly with detailed report.

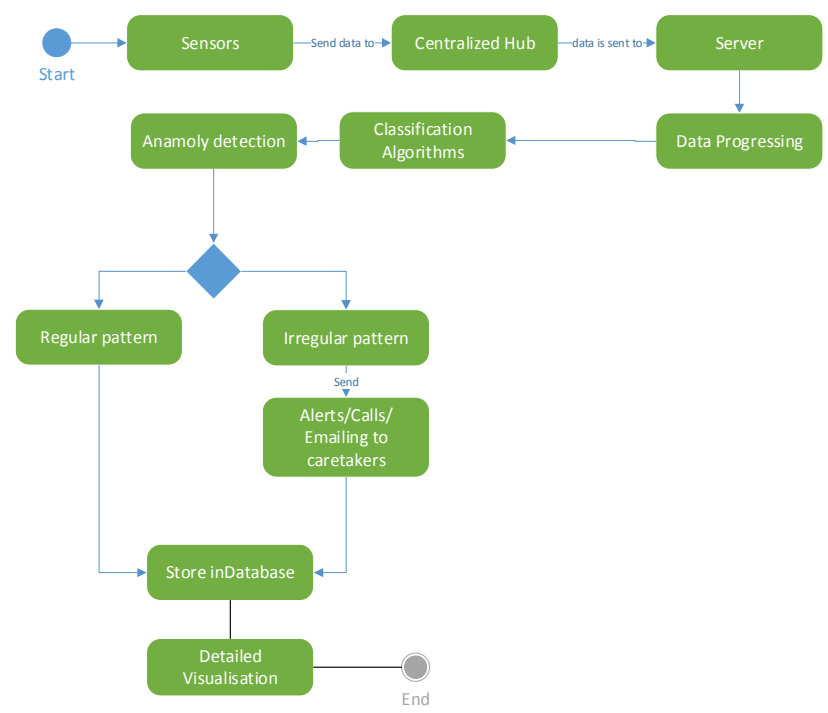

Fig. 4. Activity diagram of the system 


\section{APPLICATION FEATURES}

\section{A. Sensor location Tracker}

The sensor location tracker feature enables user to select sensor name and returns tree view map of the sensor located in the house. This interface is apartment dependent so when a user registers and sensors are installed the sensor location is obtained and configured in the system for that user.

\section{B. Dashboard}

Fig. 5. displays dashboard of the smart home application which the user can view after authentication. The top right side of the application displays user account details, notifications, user statistics and personal details. The right side of the application displays the menu that leads to various features of the application. Dashboard provides concise information such as the first row displays information about the temperature, humidity and current location of the elderly person. Also indicates whether the person has locked or not locked the main door of the house giving an insight about the environment within the home without being physically available. The status for each data about when it was last retrieved is being displayed in the image in each box. Other part of the dashboard displays summary of activities that an elderly person performs, their profile details as well as the activity check list.

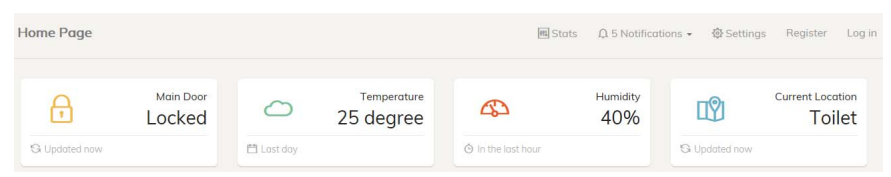

Fig. 5. System Dashboard

\section{Activity Checklist}

Activity Checklist gives a quick summary of activities that an elderly person usually performs in a day. Each activity has been given a specific icon to represent activity along with its name. If the activity has been performed by the elderly person for the day, it gives green colored feedback along with its start and end time for the latest time it was performed. If the activity has not yet been performed it gives feedback in red and shows it is awaiting action.

\section{User Profiling \& Authentication}

This feature profiles ADL of an elderly person who is being monitored for each calendar day. It also visualizes his/her personal details such as height, weight, age, address and other necessary details.

\section{E. Activity Summarization}

Activity summarization gives detailed summary of activities performed in various parts of the house allowing user to view details as per location/room.

The Fig. 6. displays number of times sensor was activated and deactivated for the selected date. Example Medicine cabinet whose sensor is activated 8 times in a day meaning the medicine cabinet was opened and closed (single activity) about
4 times in a day for sensor Id 57 as displayed in the image from the system.

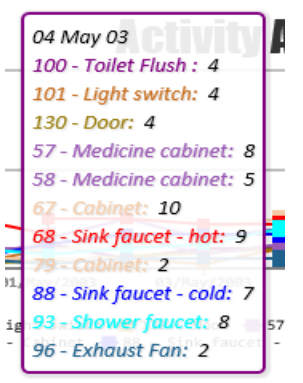

Fig. 6. Activity Summarization chart of a day

When a single activity is performed by a user, the sensor can have multiple readings i.e. one activity is broken down into chunks. Table IV displays this concept retrieved from the feature of the system where the first row is treated as parent data and the rest rows are considered as children data representing data of a person who starts an activity "Bathing" at 7:00 am and ends at 07:30 am. This feature summarizes the activity which involves multiple readings of a sensor that can be comprehended as shampooing or applying soap in between those intervals.

TABLE IV. ACTIVITY SUMMARIZATION TABLE

\begin{tabular}{|c|c|c|}
\hline Activity Details & $\begin{array}{c}\text { Sensor } \\
\text { Activation }\end{array}$ & $\begin{array}{c}\text { Sensor } \\
\text { Deactivation }\end{array}$ \\
\hline $\begin{array}{l}\text { Activity: Bathing, } \\
\text { Sensor on Shower faucet }\end{array}$ & 07:00:07 & 07:10:03 \\
\hline Dated: 07/04/2003 & 07:12:01 & 07:17:01 \\
\hline $\begin{array}{l}\text { Activity Start Time: 07:00:07 } \\
\text { Activity End Time: 7:30:10 }\end{array}$ & 07:20:11 & $7: 30: 10$ \\
\hline
\end{tabular}

Fig. 7. gives full overview of average activity performed on each day, where $\mathrm{x}$ axis represents dates on which activities were performed and $\mathrm{y}$ axis represents the count of activities that is how many times an activity was performed.

\section{Bathroom Activity Chart Activities Count per day}

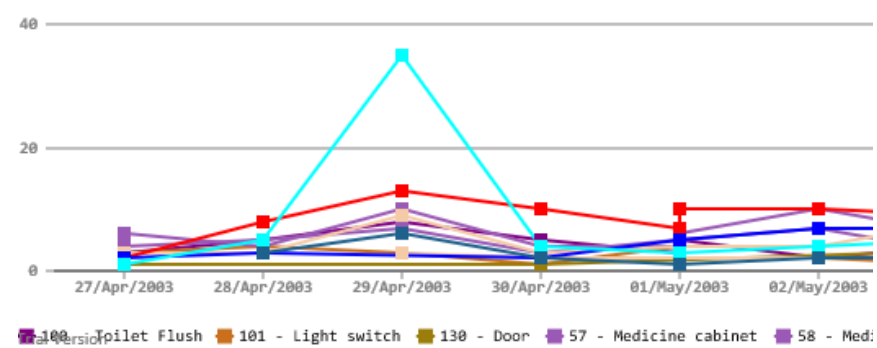

Fig. 7. Activity Chart 
The bottom line in Figure represents different colors for each house hold item where sensor have been placed such as shower facet, Medicine cabinet etc. From the Fig. 7, the pattern of activities can be seen and irregularity on specific dates can easily be visualized. This makes it easier to see that on a specific date there was some odd occurrence for a specific household chore.

\section{F. Pie Chart summary}

The pie chart in Fig. 8 gives ratio of time spent on each activity in a day by an elderly person. Ratio is calculated by dividing time spent on single activity by total time to show how much an elderly person spends on each activity in the whole day.
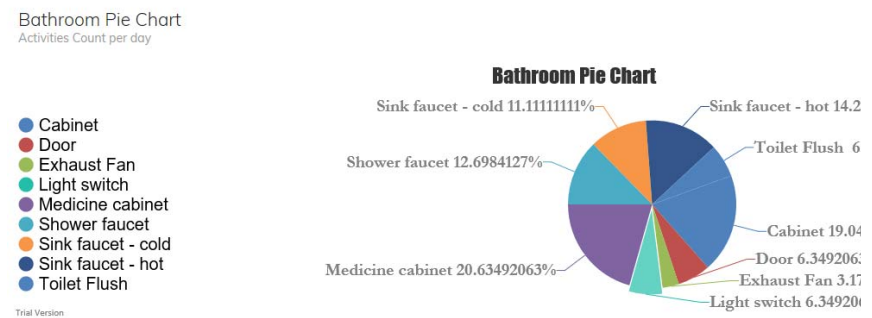

Fig. 8. Activity Pie Chart

\section{G. KNN anomaly prediction}

The dataset provided was divided into training and test data to detect anomaly. Activities were divided into two classes namely good/regular and bad/irregular. The test data is using $\mathrm{k}$ fold technique to generate different test sets in iteration along with training data. When $\mathrm{KNN}$ is applied it predicts whether the class belongs to regular or irregular. This classifier was chosen as it suits best for the criteria regarding this project as data with regular timing has been provided along with irregular data. KNN is a simple classifier and works well to classify data in categories with lower dimensions efficiently for this system. Table V shows pseudo code implementation of KNN in the system.

TABLE V. PSEUDO CODE FOR KNN

\begin{tabular}{|ll|}
\hline \multicolumn{2}{|l|}{ Pseudo code for $\boldsymbol{K N N}$ ) } \\
\hline 1. & Input: Training data, Test data. \\
2. & Output: Test data with predicted group Type. \\
3. & SELECT ADL data from table \\
4. & CACULATE SQRT ((POWER (Training Hour - Test Hour), 2) + \\
& (POWER (Training Minutes - Test Minutes),2) + (POWER \\
& (Training Seconds - Test Seconds),2)) \\
5. & ORDER data by Euclidean distance calculated in Ascending order \\
\hline
\end{tabular}

\section{H. Performance of Algorithm}

To evaluate the KNN classifier, $10 \mathrm{k}$-fold cross validation method for evaluation was used. The dataset was divided into test and training data where test data contains 10 data rows while the rest is treated as training set. The training data sample and test data predicted can be shown in the Fig. 9. taken from the application which presents the data predicted accurately for activity "Bathing" where sensor is installed on shower faucet.

\begin{tabular}{|c|c|c|c|c|}
\hline \multicolumn{5}{|c|}{ Training Data } \\
\hline SensorCode & ActivityDate & StartTime & EndTime & GroupType \\
\hline 93 & 31/03/2003 12:00:00 AM & 12:04:08 & 12:12:00 & Good \\
\hline 93 & 30/03/2003 12:00:00 AM & 08:14:13 & 08:14:40 & Good \\
\hline 93 & 30/03/2003 12:00:00 AM & 07:39:44 & 07:40:37 & Good \\
\hline 93 & 30/03/2003 12:00:00 AM & 07:38:46 & 07:38:55 & Good \\
\hline 93 & 30/03/2003 12:00:00 AM & 07:38:12 & 07:38:27 & Good \\
\hline 93 & 29/03/2003 12:00:00 AM & 16:11:34 & 16:11:35 & Good \\
\hline 93 & 29/03/2003 12:00:00 AM & 16:11:30 & 16:11:31 & Good \\
\hline 93 & 29/03/2003 12:00:00 AM & 16:11:26 & 16:11:27 & Good \\
\hline 93 & 29/03/2003 12:00:00 AM & 16:11:21 & 16:11:23 & Good \\
\hline \multicolumn{5}{|l|}{ Test Data } \\
\hline SensorCode & ActivityDate & StartTime & EndTime & GroupType \\
\hline 93 & 31/03/2003 12:00:00 AM & 09:11:06 & 09:13:49 & Good \\
\hline 93 & 29/03/2003 12:00:00 AM & 16:03:00 & 16:05:04 & Good \\
\hline 93 & 07/04/2003 12:00:00 AM & 07:18:35 & 07:24:04 & Good \\
\hline 93 & 31/03/2003 12:00:00 AM & 08:58:16 & 09:11:05 & Good \\
\hline 93 & 11/04/2003 12:00:00 AM & 19:33:58 & 19:37:47 & Good \\
\hline 93 & 01/04/2003 12:00:00 AM & 08:47:52 & 11:50:42 & Bad \\
\hline 93 & 29/03/2003 12:00:00 AM & 16:05:05 & 16:05:11 & Good \\
\hline 93 & 09/04/2003 12:00:00 AM & 19:51:35 & 20:00:13 & Good \\
\hline
\end{tabular}

Fig. 9. Sample Training and Test data after KNN Application

\section{VIII.CONCLUSION \& FUTURE WORK}

In this paper, we have presented a design and implementation of a system that could monitor health conditions of elderly person living alone using sensors in unobtrusive manner. Allowing caretakers or loved ones to be in touch and monitor health and environment status remotely. The main purpose of smart homes for elderly was to allow non-invasive and unobtrusive monitoring without affecting their dignity while being at comforts of their homes. For future work, the system currently caters single person's activities which could widely differ if multiple elderly persons live in a smart home. Along with that extending this system to gather data on Realtime basis from an elderly home with monitoring anxiety, depression and other such mental health factors could extend the features of the system.

\section{REFERENCES}

[1] AGE UK (2017). The UK's largest charity working with older people. Available at: https://www.ageuk.org.uk.

[2] Alzheimer's Society, Dementia UK. (2017). Available at: https://www.alzheimers.org.uk/info/20091/what_we_think/93/demograp hy [Accessed 30 Nov. 2017].

[3] Mahoney, E., \& Mahoney, D. (2010). Acceptance of Wearable Technology by People with Alzheimer's Disease: Issues and Accommodations. American journal of Alzheimer's disease and other dementias. Available at http://dx.doi.org/10.1177/1533317510376944

[4] F. Portet, M. Vacher, C. Golanski, C. Roux \& B. Meillon (2013). Design and evaluation of a smart home voice interface for the elderly: Acceptability and objection aspects. Personal and Ubiquitous Computing.. doi:10.1007/s00779-011-0470-5. 
[5] N. K. Suryadevara \& S. C. Mukhopadhyay (2015). Smart homes: design, implementation and issues. doi:10.1007/978-3-319-13557-1.

[6] A. Wilde, O. Ojuroye \& R. Torah (2015) Prototyping a voice-controlled smart home hub wirelessly integrated with a wearable device. 9th International Conference on Sensing Technology (ICST), doi:10.1109/ICSensT.2015.7438367

[7] D. Poulson, C.A. Nicolle \&. M. Galley (2002). Review of the Current Status of Research on Smart Homes and Other Domestic Assistive Technologies in Support of the TAHI Trials. Loughborough: Loughborough University.

[8] M.J. Rantz, R.T. Porter, D. Cheshier, D. Otto, C.H. Servey, R.A. Johnson, M. Aud, M. Skubic, H. Tyrer, Z. He, G. Demiris, G. L. Alexander, and G. Taylor (2008). TigerPlace, A State-Academic-Private Project to Revolutionize Traditional Long-Term Care. doi: $10.1080 / 02763890802097045$

[9] H. Noguchi, T. Mori, \& T. Sato (2002). Construction of network system and the first step of summarization for human daily action data in the sensing room. doi: 10.1109/KMN.2002.1115157

[10] D.J. Cook (2012). Learning Setting-Generalized Activity Models for Smart Spaces. (27)1. 32-38. Available at $\mathrm{http}: / /$ ieeexplore.ieee.org/stamp/stamp.jsp?arnumber $=5567086$

[11] P. Rashidi \& D.J. Cook (2009) Keeping the Resident in the Loop: Adapting the Smart Home to the User. doi: 10.1109/TSMCA.2009.2025137

[12] J. Ye, G. Stevenson \& S. Dobson (2016). Detecting Abnormal Events on Binary Sensors in Smart Home Environments. Pervasive and Mobile Computing, 33, 32-49. doi:10.1016/j.pmcj.2016.06.012

[13] M. R. Alam, M.B.I. Reaz \& M.A.M Ali (2012). A Review of Smart Homes: Past, Present, and Future. doi:10.1109/TSMCC.2012.2189204

[14] K. Peetoom, M. Lexis, M. Joore, C. Dirksen \& L. De Witte (2015). Literature review on monitoring technologies and their outcomes in independently living elderly people. In Disability and Rehabilitation: Assistive Technology. doi:10.3109/17483107.2014.961179

[15] D. Seo, B. Yoo \& H. Ko (2016). Data-Driven Smart Home System for Elderly People Based on Web Technologies. Distributed, Ambient and Pervasive Interactions. doi:10.1007/978-3-319-39862-4 12

[16] A. García-Hernando, Q. Ni \& I. Pau (2015). The Elderly's Independent Living in Smart Homes: a Characterization of Activities and Sensing Infrastructure Survey to Facilitate Services Development. Sensors. doi: $10.3390 / \mathrm{s} 150511312$

[17] R. Lun, C. Gordon \& W. Zhao (2016). Tracking the Activities of Daily Lives: An Integrated Approach. Future Technologies Conference. 466475.

[18] S. Chernbumroong, S. Cang \&.H. Yu (2014). A practical multi-sensor activity recognition system for home-based care. Decis. Support System. doi:10.1016/j.dss.2014.06.005

[19] Lara, O.D., Pérez, A.J., Labrador, M.A. \&., Posada, J.D. (2012). A human activity recognition system based on acceleration and vital sign data. Persuasive and Mobile Computing (8)5. 717-729. doi:10.1016/j.pmcj.2011.06.004

[20] Suryadevara, N., Mukhopadhyay, S.; Wang, R \&.; Rayudu, R. (2013). Forecasting the Behaviour of an Elderly Using Wireless Sensors Data in a Smart Home. Engineering Applications of Artificial Intelligence. (26)10. 2641-2652. . doi:10.1016/j.engappai.2013.08.004

[21] Zhuang, X., Huang, J., Potamianos, G \&., Hasegawa-Johnson, M. (2009). Acoustic fall detection using gaussian mixture models and GMM supervectors. In Proceedings of the IEEE International Conference on Acoustics, Speech and Signal Processing. doi:10.1109/ICASSP.2009.4959522

[22] Phua, C., Foo, V., Biswas, J., Tolstikov, A., Maniyeri, J., Huang, W., That, M., Xu, D \&.; Chu, A. (2009). 2-Layer Erroneous-Plan Recognition for Dementia Patients in Smart Homes. doi: $10.3390 / \mathrm{s} 131115434$

[23] Ordóñez, F., De Toledo, P \&., Sanchis, A. (2014). Sensor-Based Bayesian Detection of Anomalous Living Patterns in a Home Setting. doi:10.1007/s00779-014-0820-1

[24] Munguia Tapia, E., Intille, S., \& Larsson, K. (2004). Activity Recognition in the Home Using Simple and Ubiquitous Sensors. Available at $\quad$ at
files/TapiaIntilleLarson04.pdf $/ /$ web.media.mit.edu/ intille/papers-

[25] Munguia Tapia, E. (2003). Activity Recognition in the Home Setting Using Simple and Ubiquitous Sensors. Available at http://courses.media.mit.edu/2004fall/mas622j/04.projects/home/Tapia0 3.pdf

[26] T. W. H. Nelson, L. Kam-Yiu, N. K. Y. Joseph, H. Song and P. Ioannis (2017). Tracking Indoor Activities of Patients with Mild Cognitive Impairment Using Motion Sensors. In 31st International Conference on Advanced Information Networking and Applications (AINA). doi: 10.1109/AINA.2017.23

[27] A. Wilde (2011) Activity Recognition for motion-aware pervasive systems. University of Fribourg (Switzerland), Department of Informatics, Masters Thesis, 104pp. https://eprints.soton.ac.uk/272433/

[28] S. Katz \& C.A. Akpom (1976). A measure of primary sociobiological functions. doi:10.2190/UURL-2RYU-WRYD-EY3K

[29] Lawton, M \&., Brody, E. (1970). Assessment of Older People: Selfmaintaining and Instrumental Activities of Daily Living. doi:10.1093/geront/9.3_Part_1.179

[30] Meditskos, G., Dasiopoulou, S \&., Kompatsiaris, I. (2015). A Knowledge-Driven Framework for Context-Aware Activity Recognition Combining SPARQL and OWL 2 activity patterns. doi:10.1016/j.pmcj.2015.01.007

[31] Okeyo, G., Chen, L \&., Wang, H. (2014). Combining ontological and temporal formalisms for composite activity modelling and recognition in smart homes. doi:10.1016/j.future.2014.02.014

[32] S. H. Shah, A. Iqbal \& S. S. A. Shah (2013). Remote health monitoring through an integration of wireless sensor networks, mobile phones amp; Cloud Computing technologies. doi:10.1109/GHTC.2013.6713719

[33] C. Chen \& C. Pomalaza-Raez (2009). Design and Evaluation of a Wireless Body Sensor System for Smart Home Health Monitoring. Available from http://dx.doi.org/10.1109/GLOCOM.2009.542547

[34] Zheng, Yali., Ding X., Poon, C., Lo, B., Zhang, H., Zhou, X., Yang, G., Zhao, N \&., Zhang, Y. (2014). Unobtrusive Sensing and Wearable Devices for Health Informatics. Transactions on Biomedical Engineering. Doi:10.1109/TBME.2014.2309951

[35] Pires, I.M., Garcia, N.M., Flórez-Revuelta, F.: Multi-sensor data fusion techniques for the identification of activities of daily living using mobile devices. In: Proceedings of the ECMLPKDD 2015 Doctoral Consortium, European Conference on Machine Learning and Principles and Practice of Knowledge Discovery in Databases, Porto, Portugal (2015)

[36] National Instruments. (2017). Data Acquisition - National Instruments. Available at: http://www.ni.com/data-acquisition/what-is/

[37] Tahani, A \&., Sreela, S. (2016) Intelligent imputation technique for missing values, Advances in Computing Communications and Informatics (ICACCI). Doi:10.1109/ICACCI.2016.7732423

[38] Yoshizawa, M., Takasaki, W \&., Ohmura, R. (2013). Parameter Exploration for Response Time Reduction in Accelerometer-Based Activity Recognition. Doi:10.1145/2494091.2495986

[39] P. Li, Z. Chen, L. T. Yang, Q. Zhang and M. J. Deen (2017). Deep Convolutional Computation Model for Feature Learning on Big Data in Internet of Things. doi:10.1109/TII.2017.2739340

[40] X. Wang, L. T. Yang, J. Feng, X. Chen and M. J. Deen (2016). A Tensor-Based Big Service Framework for Enhanced Living Environments. doi:10.1109/MCC.2016.130

[41] Q. Zhang, L. T. Yang, Z. Chen, P. Li and M. J. Deen (2017). Privacypreserving Double-projection Deep Computation Model with Crowdsourcing on Cloud for Big Data Feature Learning Available at https://doi.org/10.1109/JIOT.2017.2732735 